\title{
Protein crosslinks influence food digestion
}

\section{Opinion}

Enzymatic crosslinking is increasingly applied to confer specific properties to different proteins and, consequently, to food products of which they are components. Among the most investigated enzymes, transglutaminases (in particular the microbial isoform, mTG) and various oxidative biocatalysts are having special attention by food biotechnology researchers. mTG catalyzes isopeptide bond formation among protein molecules, leading to inter-molecular crosslinks and being able to produce both homo- and hetero-polymers. Its peculiar properties, such as the calcium independency, the broad substrate specificity, the stability over a wide range of temperatures and $\mathrm{pH}$ values, make such enzyme an effective tool to modify the characteristics of many protein-based foods. In fact, it has been demonstrated that the firmness, viscosity, elasticity and water-binding capacity of different kinds of meat, fish, wheat, as well as various dairy products, are influenced by $\mathrm{mTG}$ treatment changing their texture and some functional properties. In the cereal sector mTG has been utilized to improve the strength of weak and low-quality flour, improving the texture and volume of bread and the texture of pasta after cooking. The enzyme can amend also the gluten networking properties and, consequently, it can improve the baking grade of the low-quality gluten. Furthermore, since the introduction of covalent bonds into protein gel network is known to increase the gel firmness, better structural and sensory properties of acid milk gels have been obtained in yogurt industrial production. $\mathrm{mTG}$ has been also used to strengthen the texture of homogenized sausages made with pork, beef or poultry meat, as well as it was also successfully exploited in restructuring fish cuts. The wide use of the enzyme in the food sector raised questions about the possible effects of the formed isopeptide crosslinks on protein digestibility. In fact, most of times mTG treatment was shown to influence the digestion of several foods as demonstrated by in vitro studies carried out under physiological conditions. Gastric and duodenal digestion experiments were performed in order to study the proteolysis of edible hydrocolloid films prepared by using carbohydrates and mTG-modified proteins. In particular, it has been demonstrated that Citrus pectins and the mTG-treated phaseolinbased films were more easily digested with respect to the films prepared in the absence of the enzyme, encouraging an application of the proposed biomaterials as food coatings. On the other hand, mTG-catalyzed crosslinking of milk $\beta$-casein was demonstrated to make this protein more resistant to the gastric pepsin digestion when compared to the uncrosslinked $\beta$-casein. Moreover, both gastric and

\author{
Volume 2 Issue 4 - 2017
}

\author{
Valeria L Giosafatto C, Raffaele Porta \\ Department of Chemical Sciences, University of Naples \\ "Federico II", Italy
}

\begin{abstract}
Correspondence: Raffaele Porta, Department of Chemical Sciences, University of Naples “Federico II”, Naples, Italy,
\end{abstract} Email raffaele.porta@unina.it

Received: February 23, 2017 | Published: March 03, 2017

duodenal digestion, carried out in vitro under simulated physiological conditions, allowed to evaluate the effect of the enzyme on the proteolysis of ovalbumin, the most abundant protein occurring in egg white. In particular, ovalbumin was modified via mTG following heat denaturation to obtain the completely or partially unfolding of the protein and to increase the extent of the enzymatic crosslinking. In this case the introduction of isopeptide bonds was shown to confer to the protein an increased resistance to be digested in the gastric environment. In addition, the performed experiments showed that a certain amount of the produced protein polymers persisted even in the duodenal environment, as demonstrated by the observed decrease in trypsin and chymotrypsin proteolysis. Similar results were obtained when the in vitro digestibility of soy and bean flour was analyzed. In fact, the covalent crosslinking of both soy and bean flour proteins resulted in their decreased digestion, especially when pepsin-catalyzed proteolysis was taken into consideration. In all instances, the tight and compact protein structure due to enzymatic crosslinking most likely explains the reduced observed digestion rate. These findings might have high impact on the development of novel functional food products able to enhance satiety, even though the crosslinked proteins, most of times digested more slowly, exhibit a nutritional value and an aminoacid bioavailability similar to the ones of untreated proteins.

\section{Acknowledgements}

None.

\section{Conflict of interest}

The author declares no conflict of interest. 